\title{
CORRECTION
}

\section{Correction to: Real-World Outcomes in Historically Underserved Patients with Chronic Hepatitis C Infection Treated with Glecaprevir/Pibrentasvir}

\author{
Alessio Aghemo - Yves Horsmans - Stefan Bourgeois - Mark Bondin • Michael Gschwantler - Harald Hofer • \\ Nasser Semmo · Francesco Negro - Zhenzhen Zhang · John Marcinak · Ella Veitsman · Rawi Hazzan · \\ Konstantinos Mimidis · Ioannis Goulis · Nuno Marques · Robert Flisiak · Wlodzimierz Mazur · Carlos Roncero · \\ Fiona Marra $\cdot$ Georges Philippe Pageaux $\cdot$ Tarik Asselah $\cdot$ Pietro Lampertico
}

Published online: September 16, 2021

(C) The Author(s) 2021

Correction to: Infect Dis Ther

https://doi.org/10.1007/s40121-021-00455-1

In the Original publication of the article the key summary points were incorrectly published.

This has been corrected here.

The original article can be found online at https://doi. org/10.1007/s40121-021-00455-1.

\section{A. Aghemo}

Department of Biomedical Sciences, Humanitas

University, Rozzano, Italy

\section{Y. Horsmans}

Cliniques Universitaires Saint-Luc, UCL, Brussels, Belgium

S. Bourgeois

Stuivenberg ZNA, Antwerp, Belgium

M. Bondin $\cdot$ Z. Zhang $\cdot$ J. Marcinak AbbVie Inc, North Chicago, IL, USA

\section{Gschwantler}

Department of Internal Medicine IV, Wilhelminenspital, and Sigmund Freud University, Vienna, Austria

\section{Key Summary Points}

\section{Why carry out this study}

The global elimination of HCV infection is a possibility with the availability of shortcourse pangenotypic direct-acting antivirals, however rapid expansion of treatment to historically marginalized patients is necessary to achieve the goal of elimination.
H. Hofer

Department of Internal Medicine, Gastroenterology and Hepatology, Klinikum Wels-Grieskirchen, Wels, Austria

\section{N. Semmo}

Department of BioMedical Research, Hepatology, Inselspital, University of Bern, 3010 Bern,

Switzerland

F. Negro

Division of Gastroenterology and Hepatology,

University Hospital, Geneva, Switzerland

E. Veitsman

Liver Unit, Rambam Health Care Campus, Haifa, Israel 
This multinational study presents the efficacy, safety, and patient-reported outcomes of treatment with G/P in historically underserved patient populations in real-world settings.

What was learned from the study?

Overall, G/P treatment was highly effective with a cure rate of $98 \%$ and welltolerated across a broad range of marginalized patients with chronic $\mathrm{HCV}$ and single or dual comorbidities.

Results from this real-world analysis address current gaps in clinical knowledge regarding impact on quality of life and G/P treatment led to improvements in health-related quality of life and reductions in fatigue.

R. Hazzan

Emek Medical Center, Afula, Israel

K. Mimidis

First Department of Internal Medicine, Democritus University of Thrace Medical School,

Alexandroupolis, Greece

I. Goulis

4th Department of Internal Medicine, Aristotle

University of Thessaloniki, Thessaloniki, Greece

N. Marques

Infectious Diseases Service, Hospital Garcia de Orta EPE, Almada, Portugal

R. Flisiak

Department of Infectious Diseases and Hepatology, Medical University of Białystok, Białystok, Poland

W. Mazur

Clinical Department of Infectious Diseases, Medical

University of Silesia, Katowice, Poland

C. Roncero

Psychiatry Service, University of Salamanca Health

Care Complex, Salamanca, Spain

\section{F. Marra}

Hepatology Drug Interactions Group, University of Liverpool, Liverpool, UK

\section{G. P. Pageaux}

Département Hépato-Gastro-Entérologie, Centre Hospitalier Universitaire (CHU) de Montpellier, Montpellier Cedex 5, France
These results further support the use of an 8-week short-course G/P in underserved patients including those with substance abuse and psychiatric comorbidities.

Open Access. This article is licensed under a Creative Commons Attribution-NonCommercial 4.0 International License, which permits any non-commercial use, sharing, adaptation, distribution and reproduction in any medium or format, as long as you give appropriate credit to the original author(s) and the source, provide a link to the Creative Commons licence, and indicate if changes were made. The images or other third party material in this article are

\section{T. Asselah}

Department of Hepatology, Hopital Beaujon, AP-

HP, Paris University and INSERM UMR 1149, Clichy, France

P. Lampertico

Foundation IRCCS Ca' Granda, Ospedale Maggiore Policlinico, Policlinico-Division of Gastroenterology and Hepatology-CRC 'AM and A Migliavacca' Centre for Liver Disease, Milan, Italy

P. Lampertico

University of Milan, Milan, Italy

A. Aghemo $(\square)$

Division of Internal Medicine and Hepatology, Department of Gastroenterology, Humanitas Research Hospital IRCCS, Via A. Manzoni 56, 20089 Rozzano, Milan, Italy

e-mail: Alessio.aghemo@hunimed.eu

C. Roncero

Institute of Biomedicine and School of Medicine, University of Salamanca, Salamanca, Spain

F. Negro

Division of Clinical Pathology, University Hospital, Geneva, Switzerland 
included in the article's Creative Commons licence, unless indicated otherwise in a credit line to the material. If material is not included in the article's Creative Commons licence and your intended use is not permitted by statutory regu- lation or exceeds the permitted use, you will need to obtain permission directly from the copyright holder. To view a copy of this licence, visit http:// creativecommons.org/licenses/by-nc/4.0/. 